\title{
Essais
}

ESSAIS

Revue interdisciplinaire d'Humanités

Hors-série 7 | 2022

Récits d'outre-thèse

\section{Comment les restrictions budgétaires à l'Université brisent des vies professionnelles}

Exemple à partir de ma propre trajectoire

How budgetary cuts at the University shatter professional lives

Example from my own trajectory

\section{Camille Noûs}

\section{OpenEdition}

Journals

Édition électronique

URL : https://journals.openedition.org/essais/10745

DOI : 10.4000/essais. 10745

ISSN : 2276-0970

Éditeur

École doctorale Montaigne Humanités

Édition imprimée

Date de publication : 15 avril 2022

ISBN : 979-10-970024-00-0

ISSN : $2417-4211$

Référence électronique

Camille Noûs, «Comment les restrictions budgétaires à l'Université brisent des vies professionnelles

», Essais [En ligne], Hors-série 7 | 2022, mis en ligne le 01 février 2022, consulté le 19 janvier 2023

URL : http://journals.openedition.org/essais/10745 ; DOI : https://doi.org/10.4000/essais.10745

Ce document a été généré automatiquement le 19 janvier 2023.

Tous droits réservés 


\section{Comment les restrictions budgétaires à l'Université brisent des vies professionnelles}

Exemple à partir de ma propre trajectoire

How budgetary cuts at the University shatter professional lives

Example from my own trajectory

Camille Noûs

1 Nous rêvons tous d'exercer un métier qui nous passionne. Jeune étudiant, j'ai choisi de devenir maître de conférences. J'ai effectué tout mon cursus universitaire et professionnel dans cet objectif : un master recherche avec mention dans la prestigieuse école de Sciences Po Paris, celle-là même qui produit présidents et ministres ; j'ai écrit une thèse soutenue en 2015 avec les félicitations à l'unanimité du jury; j'ai été qualifié à exercer les fonctions de maître de conférences dans trois disciplines en science politique, en sociologie et en civilisation romane; je viens de publier un livre que j'ai coécrit et un autre que j'ai co-dirigé va sortir prochainement; j'ai publié également treize articles dans des revues à comité de lecture, dont trois classés, sept chapitres d'ouvrage, quatorze articles dans des revues sans comité de lecture, organisé deux colloques internationaux et trois journées d'études; j'ai enseigné 931 heures. Et pourtant. Et pourtant, je suis au chômage depuis maintenant plus de six ans. J'ai bien eu une audition en 2019 à la Sorbonne où j'ai été classé deuxième attestant que je possède les prérequis pour devenir enseignant-chercheur titulaire à l'Université. Les rapports pour chacune de la vingtaine de candidatures que je fais chaque année depuis cinq ans soulignent un "parcours brillant», de "grande qualité », le "dynamisme de l'activité de recherche » et pourtant, à l'exception de ce comité de sélection de la Sorbonne, tous les autres depuis maintenant cinq ans refusent de m'auditionner: pas assez de formation en langues, pas assez dans le profil du poste, pas assez de publications classées... Chacun a sans doute une bonne raison pour ne pas m'auditionner ou plutôt tous ont en dernière instance, la même raison, trop de candidats pour pas assez de 
postes. Trop de jeunes docteurs, une centaine en moyenne pour chaque recrutement, aux profils toujours plus brillants pour des emplois toujours plus rares malgré le nombre d'étudiants qui augmente, un déséquilibre qui conduit forcément à une sélection toujours plus délirante.

Et pourtant, je tente de jouer le jeu de ce milieu professionnel toujours plus sélectif, je travaille à plein temps, les soirs, les weekends, avec des statuts toujours plus variés (salarié dans certains établissements privés, auto-entrepreneur dans d'autres établissements privés, vacataire à l'université). Durant l'année scolaire 2019-2020, j'ai dispensé 277 heures équivalent TD de cours (192 heures à l'université, 85 heures dans différents établissements privés de l'enseignement supérieur), soit $144 \%$ d'un service d'un maître de conférences titulaire, en ne comptant que mes activités d'enseignement, sans parler de mes activités de recherche. J'ai organisé un colloque international, publié un chapitre d'ouvrage dans une maison d'édition prestigieuse, un ouvrage coécrit... Près d'une fois et demi le travail d'un enseignant titulaire donc, mais pas la même paie évidemment, et de loin. Si les entreprises privées payent mensuellement comme le prévoit le Code du travail depuis 1971, les Universités françaises se permettent de payer avec plusieurs mois de retard. Ainsi, le paiement de mes cours dispensés entre septembre et décembre a été versé en février, le paiement de ceux dispensés entre janvier et mars en juillet. Mon contrat de travail, qui d'après le Code du travail doit être signé avant le début des tâches à réaliser, n'a été signé qu'en décembre, trois mois après le début de mes cours. Que se serait-il passé si j'avais eu un accident de travail entre septembre et décembre, par exemple? Je l'ignore. Les règles les plus élémentaires du droit du travail ne sont donc pas respectées à l'Université mais la précarité a évidemment surtout un objectif budgétaire. Ainsi, en réalisant donc une fois et demi un service d'enseignements de maître de conférences, j'ai gagné un peu moins que le SMIC.

3 Mon activité étant irrégulière, dépendant du calendrier scolaire, les mois où ma paie est faible ou nulle, c'est le RSA qui la complète, depuis plus de 4 ans désormais. Le RSA et son cortège de contrôles, ses rendez-vous à Pôle emploi, ses rendez-vous au Pôle Insertion du Conseil départemental aussi, des rendez-vous chronophages avec des propositions qui ne correspondent pas à mon parcours professionnel, avec des conseillers qui souvent ignorent tout de la réalité de notre milieu professionnel. Le véritable problème est que des établissements public ou privé refusent d'établir un véritable contrat de travail avec des congés payés comme l'exige le Code du travail. Ainsi, quelqu'un comme moi se retrouve à se faire financer ses mois d'inactivité par les budgets et des services normalement dévolus aux plus démunis.

\section{La perte de sens du métier d'enseignant-chercheur}

La précarité au travail a des effets destructeurs sur un individu. Le fonctionnement de l'Université a désormais ce pouvoir magique de le faire vivre. À force de travail pour suivre le rythme effréné d'une sélection sans fin, à force de statuts individualisant ma condition, je me désocialise. Je le constate, année après année, le temps consacré à ma compagne, à mes amis, à ma famille diminue peu à peu. À force d'un volume horaire d'enseignement de près d'une fois et demi supérieur à celui de mes collègues titulaires et d'une activité de recherche sans doute supérieure à celle de nombre d'entre eux, je 
me sens usé physiquement. En janvier 2020, je suis tombé malade à plusieurs reprises, signe de l'impact de ma vie professionnelle sur ma santé physique et psychique.

5 La précarité de l'ESR me désocialise, m'use physiquement, elle devrait logiquement m'inciter à me mobiliser contre ceux qui sont responsables des maux dont je souffre. Paradoxalement, ce ne sont pas les plus précaires qui peuvent se mobiliser. Précisément parce que nous sommes dans cette aliénation. Parce qu'il faut absolument prendre en charge ce nouveau cours, publier cet article supplémentaire pour avoir une chance de plus de pouvoir obtenir un poste l'année suivante, cette aliénation demeure puissante. En tant que précaire, il nous est extrêmement difficile de nous mettre en grève sans risquer d'être blacklisté l'année suivante. Dans les établissements privés au sein desquels j'ai enseigné, ce moyen d'action est tellement inconcevable qu'il aurait été assimilé à une simple fainéantise. Je n'ai même pas pu participer au mouvement naissant en mars contre la Loi de Programmation de la Recherche (LPR) qui promet de précariser encore plus des personnes comme moi, de remplacer les rares postes de maitres de conférences par des tenures tracks supprimant toute sécurité de l'emploi et nous amenant, dans le pire des cas, à des reconversions forcées à 45 ans. Et pourtant, cette mobilisation me concernerait et j'aurais tenu à y participer. Et pourtant, il ne manquera pas d'un petit porte-parole du gouvernement pour dire que les précaires sont absents de ces mobilisations, omettant de préciser que nous sommes absents par l'exploitation que l'on subit.

6 Ce métier me désocialise, me fatigue physiquement pour une reconnaissance si faible. Les conditions dans lesquelles j'enseigne, dans lesquelles nous enseignons, fait qu'il perd peu à peu toute signification. À l'Université, les enseignements sont de plus en plus un lieu de sélection. L'espoir d'une démocratisation de l'enseignement supérieur s'éloigne à mesure que les restrictions budgétaires se transforment en procédures restrictives sélectionnant les étudiants. L'objectif ultime n'est pas d'apprendre mais de passer à l'échelon supérieur malgré tout. Dans les établissements privés, la sélection n'est pas réellement une option, le paiement de frais d'inscription vaut quasiment validation du diplôme, les notes s'envolent ou les procédures de rattrapages font en sorte que les étudiants valident leur diplôme que le cours soit assimilé ou pas. Dans les deux cas, une part importante d'étudiants n'a que faire d'un cours de sciences sociales dans lequel ils ne voient aucun intérêt immédiat pour éviter le chômage de masse auquel ils sont promis. Le management et ses perspectives d'emplois en attirent plus d'un vers des bullshits jobs dont ils retireront peut-être un salaire mais si peu d'intérêt. Et comment leur en vouloir. Il existera toujours des étudiants intéressés qui donnent un peu de valeur au cours mais globalement, tout cela crée une profonde perte de sens pour l'enseignant.

7 Désocialisation, fatigue physique, perte de sens, amertume, écœurement, si j'en suis dans cette situation, j'en suis bien évidemment responsable, les articles que j'ai pu écrire auraient pu être plus brillants, mes méthodes pédagogiques plus dynamiques, j'aurais pu m'organiser mieux, dormir moins, ne pas dormir peut-être... Mais nous étions 128000 vacataires dans l'Université selon le Ministère. Il y a donc bien des responsabilités structurelles, des individus qui organisent, consciemment ou non, un système qui produit cette perte de sens, cette amertume et cet écœurement. 


\section{Les responsables de mon mal-être}

8 Je suis écœuré par ceux qui nous gouvernent. Ce sont eux qui sont responsables de l'organisation de la pénurie qui détruit l'Université comme ils détruisent l'hôpital public et l'Éducation nationale. En ces temps de pandémie, où les personnels soignants ont souffert des restrictions budgétaires dans la santé, il y a presque quelque chose d'indécent à se plaindre du fonctionnement de l'Université. Et pourtant, c'est rigoureusement le même problème de destruction lente des services publics qui crée tant de perte de sens, tant d'amertume, tant d'écœurement chez ceux qui y travaillent. Non seulement parce qu'il manque des postes mais aussi parce que la logique de restriction budgétaire pourrit tout, multiplie les tâches administratives chronophages, augmente la part de travail assurée par des travailleurs précaires comme moi sur des tâches durables.

9 Je suis écœuré par les méthodes de sélection. Cette année, j'ai appris qu'une professeure a délibérément menti à mon sujet lors d'un comité de sélection pour placer un de ses collègues et me barrer la route d'une audition. Et en même temps, comment ne pas comprendre dans un milieu où tout le monde joue sa survie qu'elle tente de placer ses proches? Un des critères de sélection souvent évoqués est celui de publication dans des revues classées et plus haut, elle est placée, mieux, c'est. Dans la pratique, il s'agit davantage de la préservation de réseaux de cooptation que d'une quelconque qualité scientifique. Plusieurs de mes articles fondés sur des enquêtes de terrain ont été refusés par l'évaluation de collègues en désaccord politique avec mon analyse. L'une d'entre elles s'est permise de faire la même chose avec plusieurs de mes collègues, utilisant sa position dans la hiérarchie pour s'assurer un monopole de ce qui doit être dit sur le terrain en question. Ces évaluations sont supposées être anonymes mais quel anonymat peut exister dans un milieu d'interconnaissance où chacun est informé des thématiques de recherche des uns et des autres? Où est la science làdedans? Ce critère de sélection est absurde pour le pire comme pour le meilleur. En somme, le critère des revues classés n'a qu'une faible valeur scientifique mais permet simplement de coopter ou de rejeter les nouveaux entrants. J'ai constaté la même pratique dans les chapitres d'ouvrage, il n'existe pas de corrélation entre scientificité reconnue de l'éditeur et niveau d'exigence à propos du texte remis. Lorsque le nombre de candidats qualifiés en science politique est de 16 par poste à pourvoir ${ }^{1}$, quel que soit le critère choisi, la sélection est forcément arbitraire.

10 Je suis écœuré par le statut des vacataires, non seulement nous sommes sous-payés, avec des mois de retard, mais le nombre d'heures pendant lesquelles nous pouvons exercer notre métier est limité pour des raisons bureaucratiques. Ce n'est pas par méconnaissance des besoins, ils sont énormes face au manque de postes. Mes collègues titulaires en viennent à me contacter pour faire des cours improbables pour lesquels je n'ai aucune compétence, que je refuse par conscience professionnelle. Si le nombre d'heures est limité, c'est que la vacation était initialement destinée à l'intervention de professionnels dans le milieu universitaire. Aujourd'hui, ce dispositif est utilisé principalement à l'emploi déguisé de docteurs sans postes dans des conditions relevant très clairement du racisme de classe, d'un apartheid anti-pauvres. Ainsi, l'Université n'« embauche » comme vacataire que des personnes ayant une activité professionnelle principale. Ce qui est absurde, car un jeune docteur comme moi a besoin de faire cours pour enrichir son CV et avoir ainsi une hypothétique chance d'être recruté. Ce qui est 
d'autant plus absurde, c'est que si j'avais une activité professionnelle principale, je ne perdrais pas mon temps à faire des cours sous-payés avec plusieurs mois de retard. Ces contraintes administratives mises en place par ceux qui gouvernent créent des difficultés kafkaïennes pour les titulaires afin de trouver des vacataires susceptibles d'assurer des cours, mais surtout pour les vacataires comme moi qui subissent des humiliations supplémentaires et ne peuvent pas exercer leur métier parce que trop pauvres. Un des moyens de contourner cette réglementation absurde est d'utiliser le statut d'auto-entrepreneur, auquel je me suis résolu. Mais là encore, il faut justifier, avis d'imposition à l'appui, de plus de 10000 euros de revenus chaque année depuis trois ans, « interdit aux pauvres ", on vous dit. J'ai été refusé dans trois Universités sur ce genre de motifs. Dans mon malheur, j'ai la chance d'avoir trouvé une législation un peu plus souple dans une quatrième Université. En 2019-2020, j'ai même pu monter jusqu'à 192 heures annuelles en présentant un avis de Cotisation Foncière des Entreprises. Pendant le confinement, la direction de l'Université s'est rendue compte que nombre de vacataires ne rempliraient plus les conditions requises en raison de la réduction de l'activité. L'arrogance du Ministère l'a conduit pendant le confinement jusqu'à imaginer de ne pas rémunérer les vacataires qui assuraient pourtant la continuité pédagogique. Il a fallu se battre pendant des semaines pour être simplement rémunéré pour le travail effectué. Ceux qui gouvernent ne nous auront épargné aucune humiliation.

11 Enfin, je suis écœuré par moi-même, par ce que je deviens. Dans ce milieu de précarité généralisée, il est difficile de ne pas voir ses collègues, quelques fois ses propres amis comme des concurrents. Lorsqu'un des rares postes est publié, il est difficile de ne pas imaginer lequel d'entre eux pourrait être celui qui pourrait potentiellement te passer devant.

J'accepte des sacrifices nombreux. Le tout pour un métier loin d'être rêvé, rémunéré bien en-deçà du niveau de qualification, pour ne pas choisir la ville où on va vivre (l'année dernière, je candidatais à des postes entre Poitiers et Mulhouse, entre Caen et Clermont-Ferrand, pratique pour maintenir une vie de couple) mais dans l'Université d'aujourd'hui, ce n'est pas suffisant. À un âge où les uns fondent une famille, les autres encore voyagent, je répète une énième fois des cours à des étudiants qui n'en ont rien à faire, je réécris un énième article sur ma thématique de recherche pour une revue ou un livre qui ne sera pas lu, quels que soient ma perte de sens, mon amertume et mon écœurement. Ce qui me désespère, c'est que je pourrai continuer longtemps à vivre du RSA, complété des quelques vacations qu'on me permet de faire, sans jamais être recruté comme titulaire, sans jamais avoir un salaire stable et que cela conviendrait très bien à ceux qui gouvernent et à ceux qui gèrent la pénurie.

13 En consultant les rapports concernant ma candidature aux différents postes de maître de conférences, je me suis rendu compte que l'on parvenait à me reprocher mon volume d'enseignement au détriment de mes recherches. Les collègues les plus bienveillants à mon égard m'ont confirmé ce critère d'appréciation, ce n'est pas la variété et la qualité des cours donnés qui sont décisifs pour l'attribution du poste de maitre de conférences, mais l'article publié dans la revue ayant le meilleur classement. On touche à l'absurde. Si, en théorie, les maîtres de conférences sont censés consacrer la moitié de leur temps à l'enseignement, l'autre moitié à la recherche, il est de notoriété publique que dans la pratique, la plupart parviennent tant bien que mal à assurer leur service d'enseignements et les croissantes responsabilités administratives 
et que le temps consacré à la recherche est réduit à la portion congrue. Cette année, j'ai donc décidé de limiter drastiquement mes enseignements. Il s'agit d'un véritable sacrifice car il s'agissait de ma seule source de revenus hors minima sociaux. Il s'agit à l'échelle de l'ensemble de l'Université d'un immense gâchis, parce qu'il y aurait besoin, encore davantage en ces temps d'enseignement à distance, de milliers de docteurs pour encadrer les étudiants.

Mon écœurement atteint désormais ses limites, je veux arrêter mais je ne sais pas quoi faire et je me désespère devant une telle absence de perspectives. J'ai 34 ans, je suis déjà trop vieux pour commencer de nouvelles études mais encore trop jeune pour me satisfaire de rester dans ce milieu professionnel bouché. En une phrase, l'écœurement, l'amertume et la perte de sens que je ressens à l'égard du métier d'enseignementchercheur fait que je ne sais pas ce que je vais faire du restant de ma vie. Depuis la pandémie, on sait que les restrictions budgétaires dans les services publics tuent, elles brisent aussi les vies ou du moins, les vies professionnelles de millions de travailleurs précaires comme moi.

\section{NOTES}

1. ANCMSP, Bilan des recrutements MCF en science politique, 2018-2019, p. 8, mis en ligne le 15 juillet 2020, consulté le 24 décembre 2020, disponible en ligne sur : https://ancmsp.com/bilan-ancmspdes-recrutements-mcf-2018-2019. 